\title{
OTIONOMICS
}

Revista de los Estudios de Economía y Empresa

Dossier «Redes sociales, economía y empresa»

MÁS ALLÁ DEL MARKETING

\section{Las oportunidades de las redes sociales para las pymes}

\section{Monsterrat Peñarroya Earell}

Consultora de los Estudios de Economía y Empresa (UOC). Directora de Quadrant Alfa

RESUMEN En la actualidad, las redes sociales se han convertido en una de las principales herramientas de marketing de las empresas, pero aparte de ser una excelente herramienta para gestionar la relación entre cliente y empresa, estas instituciones las utilizan también para muchos otros propósitos. En este artículo se analizan estos otros usos.

PALABRAS CLAVE redes sociales, medios Sociales, e-learnig, pyme

\section{Social Media Opportunities for SMES}

ABSTRACT Nowadays, social media have become a major marketing tool for businesses. But in addition to being an excellent tool for managing the relationship with customers, social media are also used by companies for many other purposes. This article analyses these other uses.

KEYWORDS social networks, social media, eLearning

\section{Introducción}

Las redes sociales son una de las herramientas más potentes a nivel de comunicación a través de la web 2.0 y están demostrando que a nivel empresarial son una herramienta útil en muchos otros ámbitos. Encuestas a usuarios y estudios diversos indican que, cada vez más, las redes sociales son utilizadas para temas relacionados con las actividades profesionales de las personas, además de dar a conocer productos o desarrollar la relación con clientes y posibles clientes (Fundación Telefónica, 2014).

Algunas de estas redes, como Facebook por ejemplo, son tan utilizadas como plataforma online que en ciertos entornos prácticamente todo el mundo tiene una cuenta en alguna de ellas o en varias, así que no hay curva de aprendizaje en el uso de sus funcionalidades ni tampoco necesidad de conocer un nuevo entorno para ciertas actividades o para la relación entre diferentes colectivos. De ahí que muchas empresas hayan adoptado este tipo de redes para otros usos distintos al marketing. Y no solo lo están haciendo las grandes corporaciones, también para las pymes, hacia las que se orienta este artículo, las redes sociales presentan oportunidades. 


\section{Qué redes y medios sociales son los más utilizados por las empresas españolas}

Utilizando como fuente el sitio web Alexa.com, que obtiene sus datos a través de un panel de más de 15 millones de usuarios, así como a partir de datos cedidos por ISP de todo el mundo (Alexa, 2014), el ranking actual de medios sociales es el siguiente:

\begin{tabular}{|c|c|c|c|c|}
\hline Red/Medio social & $\begin{array}{c}\text { Posición respecto } \\
\text { al resto de redes y } \\
\text { medios en España }\end{array}$ & $\begin{array}{c}\text { Posición respecto } \\
\text { al resto de redes } \\
\text { y medios en el } \\
\text { mundo }\end{array}$ & $\begin{array}{c}\text { Posición global } \\
\text { respecto a todos } \\
\text { los sitios web en } \\
\text { España }\end{array}$ & $\begin{array}{c}\text { Posición global } \\
\text { respecto a todos } \\
\text { los sitios web de } \\
\text { mundo }\end{array}$ \\
\hline Facebook & 1 & 1 & 3 & 2 \\
\hline Youtube & 2 & 2 & 4 & 3 \\
\hline Twitter & 3 & 4 & 5 & 7 \\
\hline Wikipedia & 4 & 3 & 6 & 6 \\
\hline Linkedin & 5 & 5 & 9 & 10 \\
\hline Pinterest & 6 & 6 & 17 & 23 \\
\hline Instagram & 7 & 7 & 19 & 29 \\
\hline
\end{tabular}

Fuente: Alexa (http://www.alexa.com/topsites/countries/ES) a 15 de setiembre 2014.

\section{Otros usos para las redes y los medios sociales}

El marketing es uno de los principales usos que las empresas dan a las redes sociales, pero este ha sido ampliamente tratado en trabajos y artículos publicados en este mismo medio, así que en este artículo se ha querido hacer hincapié en los otros usos que las empresas dan a estas mismas redes.

Analicemos algunos de esos usos.

\subsection{E-learning para clientes y/o empleados}

Los crecimientos en el uso del e-learning por parte de empresas e instituciones estos últimos diez años no tiene precedentes. Algunos analistas estiman que la facturación de este sector para 2014 llegará a 50.000 millones de dólares. El doble que la cifra que se alcanzó cinco años atrás (Ferriman, Justin, 2014).

El e-learning a través de las redes se realiza mediante de la creación de un curso online cuyo contenido se publica en la red o el medio social y se utiliza el mismo canal para dar soporte a la formación. Las tres redes más utilizadas para impartir formación online son Facebook, Twitter y Youtube.

En redes como Facebook se realiza a partir de grupos privados en los que el administrador invita a los participantes (en caso de realizar un curso para algún colectivo concreto) o bien se realiza a través de un grupo abierto (en caso de que se desee que cualquier persona pueda acceder al curso). Se crean subgrupos para cada materia y el administrador o el profesor publican como entrada inicial de cada subgrupo el contenido sobre el que vaya a versar la lección. A partir de ese contenido, los alumnos discuten o bien presentan sus propuestas abiertas al resto de alumnos.

En Twitter, por el contrario, lo que se suele hacer es dividir el contenido de un curso en frases de 140 caracteres que serán transformadas en tuits y que podrán ser publicadas utilizando una herramienta automática de 
publicación como Future Tweets (www.futuretweets.com) o Hot Suite (www.hotsuite.com). Para cada curso suele crearse un hashtag (\#nombredelcurso) para que de esta manera se puedan identificar todos los tuits que corresponden al contenido, y cualquier persona pueda incorporarse al curso en todo momento.

A través de Youtube el e-learning se realiza a base de vídeos de diferentes duraciones a los que los alumnos pueden acceder directamente desde esta herramienta, o bien por invitación (con login y password) en caso de que así lo desee el administrador. Una de las ventajas de este medio es que a través de la funcionalidad "notas" permite encadenar diversos vídeos y también permite crear dentro de los vídeos, índices que llevan a las distintas lecciones.

\subsection{Soporte a la formación}

En otros casos, las redes sociales sirven de soporte a los alumnos que están recibiendo formación a través de otras herramientas de e-learning o través de clases presenciales. Tanto Twitter como Facebook son utilizados con este fin.

En algunos casos, estas redes se usan por parte de los propios alumnos de forma espontánea sin ningún control de las instituciones educativas. Este es por ejemplo el caso de los numerosos grupos privados en Facebook que utilizan alumnos de la UOC. Existen grupos privados creados por los propios alumnos para cada carrera, con subgrupos para cada asignatura. En estos grupos privados, se discute sobre las PAC de cada materia, al margen de los profesores, pero con soporte real entre los mismos estudiantes. Cada semestre el administrador de cada subgrupo busca un nuevo miembro del curso siguiente que desee ser el administrador y le pasa el relevo de ese subgrupo. En carreras como la de Multimedia, el grupo de "Alumnes Grau Multimedia UOC" consta de 871 miembros y tiene creadas más de 50 subcarpetas.

\subsection{Relación entre empleados de una misma empresa o entre colectivos empresariales}

Puesto que las redes sociales son un punto de encuentro de miles de personas, es normal que algunos colectivos relacionados con actividades empresariales creen sus propias páginas en Facebook o sus propios grupos privados. Este es el caso, por ejemplo, de la sección del Sindicato Comisiones Obreras de la empresa Leroy Merlin en Facebook (ver https://www.facebook.com/CCOOLeroyMerlin)

\subsection{Head hunting y recursos humanos}

La búsqueda de empleados a través de Internet es un hecho habitual en España, aunque mayoritariamente se realiza a través de portales como Infojobs (www.infojobs.net). Pero es de uso común entre las empresas publicar sus ofertas de trabajo en redes como Linkedin (www.linkedin.com) o Xing (www.xing.com).

También en Facebook podemos encontrar ofertas de empleos en los grupos más activos de ciertos colectivos como el de "Spanish Animators" con 3.968 miembros y en cuyas páginas se publican tanto ofertas de empleo como ofertas formativas para los miembros de este colectivo.

\subsection{Fuente de ingresos alternativa}

Debido al uso masivo de los medios sociales por parte de los internautas, algunos de ellos han empezado a remunerar a las personas y a las organizaciones que crean los contenidos que dan vida a estos medios. Este es el caso de Youtube, por ejemplo, que aparte de permitir que los propietarios de vídeos incluyan anuncios en ellos y por lo tanto puedan recibir una remuneración, también remunera a los youtubers con más seguidores y con una gran audiencia. En España, esta se ha convertido en una de las principales fuentes de ingresos de muchas personas como Ruben Doblas (alias "elrubius") o Germán Garmendia (alias "holasoygerman"), ambos con millones de suscriptores y miles de millones de visionados de sus vídeos. Según fuentes como Social Blade (Social Blade, 2014) y como Business Insider (Harrison Jacobs, 2014), los ingresos estimados gracias a Youtube para cada uno de ellos supera el millón de dólares. 
También las empresas están utilizando Youtube como fuente de ingresos. Este es el caso, por ejemplo, de la editorial que publica las tiras cómicas del ilustrador Simon Tofield, que gracias a transformar las tiras en cortos de entre uno y tres minutos y publicarlos en Youtube bajo el título de "Simon's Cat" está obteniendo unos ingresos estimados en más un millón de dólares al año solo con los visionados de los vídeos.

\section{Los medios 3.0}

Si bien las redes sociales son herramientas de lo que a partir de 2004 denominamos la Web 2.0 (Tim O'Reilly, 2005), en la actualidad, catorce años después, estamos ante un nuevo cambio en Internet y nos encontramos ante las puertas de la web 3.0.

Las herramientas 3.0 son la evolución de las 2.0 pero con algunas características diferentes, como por ejemplo, la movilidad, el uso de la geolocalización, el uso de la web semántica, el uso de la realidad aumentada, entre otras (Harris, 2008).

Analizando algunos de los medios sociales 3.0 más utilizados, nos encontramos frente a WhatsApp, propiedad de Facebook desde 2013, que a pesar de su juventud, está siendo utilizada por algunas empresas tanto como herramienta de marketing (creando grupos para distintos tipos de cliente), como herramienta para incrementar la productividad empresarial gracias a la facilidad con la que se pueden coordinar equipos de personas.

\section{Algunos inconvenientes}

A lo largo de este artículo se han analizado algunos de los otros usos que se dan a las redes y a los medios sociales por parte de las empresas, pero sin duda, algunos de estos usos no son óptimos. Por ejemplo, el uso de Facebook como herramienta para el e-learning es complejo, puesto que al no tratarse de un entorno controlado, con mucha facilidad el alumno puede estar más pendiente de lo que se publica en el muro de sus amigos que en las lecturas y la participación en las discusiones del grupo de aprendizaje. Esto es un problema si tenemos en cuenta que se estima que un $40 \%$ de las empresas que forman parte de las Fortune 500 está utilizando herramientas de e-learning para sus empleados, en horas de trabajo como herramienta para incrementar su productividad, y por lo tanto, la competitividad de la empresa (Ferriman, 2014). Así que no todos los usos que se han comentado a lo largo de este artículo son los más adecuados para cada una de las redes sociales y los medios que se han analizado.

\section{Conclusiones}

Las redes sociales son una excelente herramienta de marketing para las empresas, aunque debido a su uso masivo por parte de los internautas se les puede dar otros usos empresariales, con resultados excelentes en la mayor parte de los casos. Algunos de estos usos son el e-learning o el soporte al mismo, la búsqueda de nuevos empleados, una fuente de ingresos alternativa, entre otros.

Puesto que estos usos en algunos casos son fruto de la espontaneidad de los propios usuarios y en otros no son $100 \%$ efectivos, es necesario analizar con más profundidad la idoneidad de cada uno de ellos para los objetivos que se desea alcanzar a nivel empresarial. 


\section{Bibliografía}

ALEXA (2014). "The Alexa Rank". Ranking mundial. About Alexa Internet. http://www.alexa.com/.

FERRIMAN, J. (2014). "The Explosive Growth of eLearning." Learn Dash. http://www.learndash.com/the-explosive-growth-of-elearning/.

FUNDACIÓN TELEFÓNICA. La Sociedad de La Información En España 2013. 2013th ed. Sociedad de La Información. Madrid: Fundación Telefónica.

HARRIS, D. (2008). Web 2.0 Evolution into the Intelligent Web 3.0: 100 Most Asked Questions on Transformation, Ubiquitous Connectivity, Network Computing, Open Technologies, Open Identity, Distributed Databases and Intelligent Applications. Australia: Emereo Pty Ltd.?].

HARRISON J. (2014). "Ranking of Youtube Biggest Stars." Business Insider. http://www.businessinsider.co.id/ richest-youtube-stars-2014-3/\#.VCsOgvl_uSo.

O'REILLY, T. (2005). "What Is Web 2.0 - Design Patterns and Business Models for the Next Generation of Software." O’Reilly. http://oreilly.com/web2/archive/what-is-web-20.html.

SOCIAL BLADE. (2014). "Youtube Statistics for Elrubiusomg”. Social Blade. http://socialblade.com/youtube/user/ elrubiusomg.

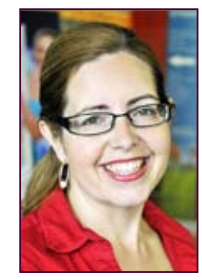

\section{Montserrat Peñarroya i Earell Consultora de los Estudios de Economía y Empresa (UOC). Directora de Quadrant Alfa}

Directora del Instituto Internacional para la Investigación de la Sociedad de la Información y el Conocimiento (3iSIC) y directora de Quadrant Alfa, empresa especializada en formación e investigación en marketing digital. Socia fundadora y directora general de Comercio Digital, S. A., empresa de Grupo Intercom que engloba, entre otros, el mayor mercado online B2B de España: SoloStocks.com, y socia fundadora de ALT64, empresa dedicada a la comercialización de herramientas orientadas a facilitar el marketing digital y la usabilidad. CEO de la editorial digital Noticias Online, que edita publicaciones como Noticias.com y que pertenece al Grupo Intercom. Profesora de Marketing Digital y de eBusiness, en másteres y posgrados de diversas universidades. Además de la UOC, colabora con la UAB, la UB y la Pompeu Fabra, entre otras. Es autora del libro Marqueting Electrònic per a Turisme Cultural de la Editorial Vitel·la.

Los textos publicados en esta revista están -si no se indica lo contrario- bajo una licencia Reconocimiento-Sin obras derivadas 3.0 España de Creative Commons. Puede copiarlos, distribuirlos y comunicarlos públicamente siempre que cite su autor y la revista y la institución que los publica (autoría, nombre de la revista, institución editora); no haga con ellos obras derivadas. La licencia completa se puede consultar en http://creativecommons.org/licenses/by-nd/3.0/es/deed.es.

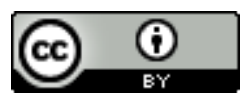

